\title{
Repercussão da monitorização fetal intraparto sobre os índices de operação cesariana
}

Impact of intrapartum fetal monitoring on cesarean section rates

Edson N. Morais, Patrícia Spara, Fabrício M. Farias

\begin{abstract}
RESUM0
A monitorização fetal eletrônica (MFE) tem sido o método mais amplamente utilizado para a vigilância fetal direta, especialmente durante o trabalho de parto. Na tentativa de elucidar o efeito da MFE sobre os índices de cesárea (IC), um estudo retrospectivo foi realizado no Hospital Universitário de Santa Maria (HUSM). Estudamos dois grupos de pacientes perfazendo um total de 2.114 gestantes: um grupo ( $n=517)$ com MFE e outro $(n=1.597)$ com ausculta intermitente (AI). No grupo MFE observamos um IC de 38,0\%, contra 27,2\% do grupo AI. Para todas as pacientes, o IC foi de 29,9\%. O sofrimento fetal agudo foi a indicação mais comum de cesárea no grupo $\mathrm{MFE}$ (40,6\%), ao passo que a cesárea prévia foi a terceira causa (10,1\%). No grupo AI, o sofrimento fetal foi a terceira causa de cesárea (14,3\%), ao passo que a cesárea prévia foi a indicação mais comum $(32,4 \%)$. Baseados no presente estudo, acreditamos que a MFE não tem efeito, por si só, sobre as taxas de cesárea, se considerados todos os nascimentos no HUSM. Com uma educação adequada dos obstetras e uma correta interpretação dos traçados, a MFE não aumenta os índices de cesárea, ao contrário permite mais acuracidade na descrição das condições fetais intraparto.
\end{abstract}

PALAVRAS-CHAVE: Monitorização fetal. Sofrimento fetal. Cesárea.

\section{Introdução}

A operação cesariana constitui-se hoje em alguns serviços de obstetrícia em nosso meio, a principal via de parto pelas mais diferentes indicações, o que faz do Brasil um dos países com maiores índices de cesárea no mundo. Nos Estados Unidos da América do Norte, as taxas de cesárea aumentaram nas últimas duas décadas de $5 \%$ para cifras superiores a $25 \%{ }^{17}$. Dentre as principais

Departamento de Ginecologia e Obstetrícia do Centro de Ciências da Saúde da Universidade Federal de Santa Maria. Correspondência:

Edson N. Morais

Av. Borges de Medeiros 1699/604

97015-090 - Santa Maria, RS causas de cesárea citam-se a interatividade, o sofrimento fetal e a desproporção cefalo-pélvica, nem sempre nessa ordem. Dentre os fatores determinantes pelo incremento das taxas de cesáreas, a monitorização fetal eletrônica (MFE) tem sido alvo de discussão pelas diferentes autoridades científicas ${ }^{4,5,6,7}$.

A avaliação do bem-estar in utero teve, a partir da década de 60, uma contribuição significativa pelo advento da monitorização eletrônica da freqüência cardíaca fetal pelos métodos direto e indireto durante o trabalho de parto. Ainda que vários estudos tenham demonstrado uma diminuição da mortalidade perinatal com a melhoria das condições de vigilância intraparto e anteparto, esta é uma 
afirmativa não confirmada por muitos. Zalar e Quilligan ${ }^{19}$ mencionam que a monitorização fetal contínua intraparto é conhecida não somente por detectar padrões que refletem sofrimento fetal mas também por causar intervenções operatórias inapropriadas, a não ser que o $\mathrm{pH}$ fetal seja conferido diretamente do sangue capilar.

O objetivo do presente trabalho foi o de estudar retrospectivamente as repercussões do uso da cardiotocografia intraparto sobre os índices de operação cesariana no Hospital Universitário de Santa Maria (RS).

\section{Material e métodos}

Foram analisadas retrospectivamente as informações sobre 2114 gestantes em trabalho de parto atendidas no Serviço de Obstetrícia do Hospital Universitário de Santa Maria que deram à luz 2134 recém-nascidos.

Das pacientes estudadas 1597 não foram submetidas à MFE intraparto, ao passo que em 517 o método foi utilizado. As pacientes monitorizadas eram portadoras de gestações de alto risco, com idade igual ou superior a 28 semanas.

Os critérios de seleção das pacientes submetidas à MFE incluiram eliminação de mecônio e alterações da freqüência cardíaca fetal (FCF) durante o trabalho de parto, portadoras de doenças intercorrentes do ciclo gestatório (hipertensão, diabetes, nefropatias, cardiopatias) e trabalho de parto prematuro.

A FCF foi monitorizada pelo método direto, isto é, pela colocação de eletrodo em espiral de Hon tipo II no couro cabeludo fetal, após a rotura das membranas amnióticas, e com dilatação cervical superior a $2 \mathrm{~cm}$. A tocografia foi realizada pelo método indireto, pela colocação de tocotransdutor de pressão colocado na altura do fundo uterino do lado oposto ao dorso fetal.

Como critérios de sofrimento fetal agudo (SFA) foram utilizados os descritos, em 1984, por Morais e Mottecy para nivel e variabilidade da linha de base da $\mathrm{FCF}^{10}$, desacelerações $\operatorname{tardias}^{11} \mathrm{e}$ desacelerações variáveis ${ }^{12}$, isto é, taquicardia e bradiacardia sustentadas (tempo superior a 10 minutos) e de repetição; variabilidade do tipo zero de Hammacher, sustentada; desacelerações tardias numa freqüência de pelo menos $20 \%$ em relação ao número de contrações uterinas; desacelerações variáveis numa freqüência de pelo menos $25 \% \mathrm{em}$ relação ao número de contrações uterinas. Para fins de análise do bem-estar fetal, com base unicamente nas mudanças progressivas da sua FCF, foram excluídos os casos de descolamento prematuro de placenta para o diagnóstico de SFA.
Para a análise das indicações de cesárea nos grupos monitorizado e não-monitorizado, levou-se em conta apenas as três principais indicações em cada grupo, isto é, sofrimento fetal, desproporção céfalo-pélvica e interatividade uma vez que no Hospital Universitário de Santa Maria ao longo dos últimos 25 anos não têm variado, a não ser na ordem de ocorrência.

\section{Resultados}

Os resultados de 2114 parturientes estudadas encontram-se nas duas tabelas apresentadas. Os partos vaginais instrumentados foram realizados através de fórcipe e vácuoextrator, sendo que apenas $5 \%$ pelo segundo instrumento.

Na Tabela 1 encontra-se a distribuição de 2114 partos segundo a sua modalidade e a realização ou não de monitorização eletrônica intraparto. Observa-se que os partos instrumentados perfizeram $19,4 \%$ do grupo monitorizado eletronicamente, ao passo que apenas $2,3 \%$ do grupo com AI. A cesárea foi a segunda via de parto mais freqüente em ambos os grupos, sendo que no grupo com MFE foi de 38\%, ao passo que no grupo com AI foi de 27,2\%. Das 2114 gestantes, em 24,5\% a cardiotocografia foi realizada.

Tabela 1 - Distribuição de 2.114 partos consecutivos realizados no HUSM, segundo 0 método de monitorização da FCF e o tipo de parto.

\begin{tabular}{|c|c|c|c|c|c|c|}
\hline \multirow{2}{*}{$\begin{array}{l}\text { MONITORIZAÇÃO DA FCF } \\
\text { TIPO DE PARTO }\end{array}$} & \multicolumn{2}{|c|}{ M F E } & \multicolumn{2}{|c|}{ A I } & \multicolumn{2}{|c|}{ TOTAL } \\
\hline & $\mathrm{n}^{\mathrm{o}}$ & $\%$ & $\mathrm{n}^{\mathrm{o}}$ & $\%$ & $\mathrm{n}^{\circ}$ & $\%$ \\
\hline Vaginal Espontâneo & 220 & 42,6 & 1.126 & 70,5 & 1.346 & 63,7 \\
\hline Vaginal Instrumentado & 100 & 19,4 & 36 & 2,3 & 136 & 6,4 \\
\hline Cesárea & 197 & 38,0 & 435 & 27,2 & 632 & 29,9 \\
\hline TOTAL & 517 & 24,5 & 1.597 & 75,5 & 2.114 & 100,0 \\
\hline
\end{tabular}

Na Tabela 2 estão arroladas as principais indicações de cesárea entre as pacientes monitorizadas pela MFE e pela AI. Observa-se que o sofrimento fetal foi a terceira causa de cesárea entre os casos com AI, enquanto entre os monitorizados eletronicamente constituiu-se na primeira indicação do parto por via alta.

Dos 517 casos com MFE, apenas 1 óbito ocorreu intraparto, ao passo que 5 vieram a falecer durante o parto entre os casos não monitorizados pela MFE. 
Tabela 2 - Distribuição de 632 partos cesáreos realizados no HUSM, segundo o método de monitorização da FCF e as principais indicações de cesárea.

\begin{tabular}{lcccccc} 
MONITORIZAÇÃO DA FCF & \multicolumn{2}{c}{ AI } & \multicolumn{2}{c}{ MFE } & \multicolumn{2}{c}{ TOTAL } \\
INDICAÇÕES DE CESÁREA & $\mathrm{n}^{\circ}$ & $\%$ & $\mathrm{n}^{\circ}$ & $\%$ & $\mathrm{n}^{\circ}$ & $\%$ \\
\hline Sofrimento Fetal Agudo & 62 & 14,3 & 80 & 40,6 & 142 & 22,4 \\
Desproporção Cefalo-Pélvica & 71 & 16,3 & 49 & 24,9 & 120 & 19,0 \\
Cesárea Prévia & 141 & 32,4 & 20 & 10,1 & 161 & 25,5 \\
Outras & 161 & 37,0 & 48 & 24,4 & 209 & 33,1 \\
\hline TOTAL & 435 & 68,8 & 197 & 31,2 & 632 & 100,9 \\
\hline MFE: monitorização & \multicolumn{3}{c}{ AI: ausculta } \\
fetal eletrônica. & \multicolumn{3}{c}{ intermitente } & & &
\end{tabular}

\section{Discussão}

A monitorização pela ausculta intermitente do coração fetal foi um método reconhecido como satisfatório para avaliação do bem-estar fetal no passado. Na segunda metade do século métodos eletrônicos de avaliação fetal foram introduzidos na prática clínica e são atualmente largamente utilizados e valorizados, principalmente durante o trabalho de parto.

A freqüência dos partos monitorizados é variável entre os diversos autores. Discute-se se todas as pacientes devem ser monitorizadas, ou apenas as que são consideradas de risco. A incidência de partos monitorizados é, no nosso entendimento, uma questão em aberto e não encontra consenso entre os autores. Sabe-se que a MFE da FCF não é isenta de riscos, ainda que sob cuidados rigorosos. Complicações como abscessos de couro cabeludo e céfalo-hematomas, entre outras, podem ocorrer ${ }^{9}$. No material por nós estudado podemos observar que apenas $24,5 \%$ dos casos foram monitorizados pela MFE intraparto, correspondendo aproximadamente ao número de gestantes de risco que têm parto no HUSM. Boehm e cols. ${ }^{1}$ preconizam o uso liberal da MFE em pacientes de baixo e alto risco. Tutera e Newman ${ }^{18}$ referem que $98 \%$ dos partos em seu serviço são monitorizados. No Long Beach Memorial Medical Center Women's Hospital, nos Estados Unidos ${ }^{3}$, todas as pacientes são monitorizadas durante o trabalho de parto. A taxa de mortalidade intraparto nesse hospital é inferior a $1 / 1000$ e a de cesárea é de 5\%. Tais índices são conseguidos às custas de uma política ativa do serviço que inclui revisões sistemáticas de todos registros cardiotocográficos e reuniões periódicas com a equipe de centro obstétrico, incluindo pessoal de enfermagem. A discussão entre todos os envolvidos na monitorização das pacientes, confere ao grupo um alto grau de compreensão sobre o assunto e a valorização da cardiotocografia, pois os índices de cesárea e de mortalidade intraparto são considerados baixos pelos autores ${ }^{3}$. Pello e cols. ${ }^{13}$ estudando 600 pacientes em trabalho de parto inicial procuraram observar se o tempo de screening das pacientes nesse momento do parto, para a continuação ou não da monitorização, poderia repercutir sobre os resultados perinatais. Dividiram as pacientes em 5 grupos em relação ao tempo de seleção: $10,15,20,30$ e 60 minutos. No que se referiu ao modo de parto, as taxas de cesárea foram maiores naquelas em que o tempo foi de 30 e 60 minutos, particularmente por indicação de sofrimento fetal. Entendem que a monitorização eletrônica intraparto não se justifica em todas as pacientes, e que um screening no trabalho de parto inicial é importante para a seleção daquelas que efetivamente irão necessitar do procedimento por tempo prolongado.

Nosso estudo demonstrou que a incidência de cesárea foi de 38,0\% no grupo com MFE, sendo que o sofrimento fetal foi responsável por 40,6\% das indicações, constituindo-se na primeira causa da operação, ao passo que a interatividade foi a terceira causa de cesárea. Por outro lado, entre as pacientes monitorizadas pela AI, o índice de cesárea foi de $27,2 \%$, e o sofrimento fetal foi apenas a terceira causa mais freqüente da cirurgia $(14,3 \%)$. A presença de cicatriz de cesárea anterior foi a primeira causa de cesárea nesse grupo $(32,4 \%)$. A taxa de cesárea na população geral estudada correspondeu a $29,9 \%$, o que significou um aumento de apenas 2,7\% quando comparado ao grupo sem MFE.

As incidências de partos cesáreos em gestantes de grupos monitorizados eletronicamente, quando comparados a grupos com ausculta intermitente, variam de autor para autor. Taxas de cesárea de 2,2 e $2,4 \%^{7}, 6$ e $11 \%^{4}, 4$ e $10 \%{ }^{5}, 14$ e $22 \%^{14}$ de gestantes de grupos com ausculta intermitente e com MFE respectivamente, foram encontradas pelos diversos investigadores. Boehm e cols. ${ }^{1}$ não observaram diferenças entre grupos monitorizados e não monitorizados durante o trabalho de parto, em relação à incidência de partos cesáreos. O estudo compreendeu um período entre 1970 e 1979. O início da monitorização fetal intraparto ocorreu em 1973, com 100\% dos partos sendo monitorizados. Nos 3 anos que antecederam o advento da MFE em seu serviço $(70,71$ e 72) as taxas de cesárea foram de 8,9, 9,2 e 12,0\%, respectivamente. Nos primeiros 3 anos subseqüentes $(73,74$ e 75$)$ com a MFE rotineiramente utilizada, estas taxas foram de 9,1, 8,8 e $12,3 \%$ respectivamente. Para esses autores o aumento gradual dos índices de cesárea até 1979 
(17\%) se deu mais pela maior liberalização da operação para apresentação pélvica e outras distocias, do que pela MFE intraparto. Mahomed e cols. ${ }^{8}$ compararam grupos de pacientes seguidas pela ausculta intermitente com sonar Doppler, ausculta intermitente pelo estetoscópio de Pinard e monitorização eletrônica contínua da FCF. De 1255 gestantes em trabalho de parto, 318 foram monitorizadas pela MFE. Encontraram uma taxa de cesárea em $28 \%$ no grupo monitorizado eletronicamente, contra $24 \%$ e $10 \%$ nos grupos seguidos com sonar Doppler e estetoscópio de Pinard, respectivamente.

A MFE permite uma melhor acuracidade diagnóstica para as alterações da FCF intraparto, o que leva à detecção mais freqüente de sofrimento fetal agudo. Por outro lado o acompanhamento do trabalho de parto pela MFE confere ao clínico maior segurança no que diz respeito à vigilância da atividade uterina, ou seja, gestantes com cicatriz de cesárea prévia podem mais freqüentemente terem seus partos por via vaginal. Esses dados podem explicar, em parte, a inversão dos índices dessas duas indicações em nosso material, quando comparados trabalhos de parto com e sem a MFE. Tutera e Newman ${ }^{18}$ referem que a operação cesariana foi realizada pela primeira vez em 62 pacientes monitorizadas $(10,1 \%)$, número significantemente superior ao do grupo não monitorizado $(4,8 \%)$. O sofrimento fetal entre pacientes monitorizadas foi a indicação prevalente para cesárea em $51 \%$ dos casos. Referem ainda que em 107 pacientes com diagnóstico de sofrimento fetal o parto se deu por via vaginal, e imputam à MFE o fato de terem evitado a cesárea.

A mortalidade intraparto no presente estudo foi de $1 / 517$ casos $(1,9 / 1000)$ entre os fetos com $\mathrm{MFE}$, enquanto que no grupo com AI foi de 3,2/ 1000. No material de Tutera e Newman ${ }^{18}$ que compreendeu 608 pacientes monitorizadas, das quais $96 \%$ foram consideradas de alto risco, a mortalidade perinatal foi de 8/1000 nascimento e a mortalidade fetal após a admissão no serviço, de 2/1000 nascimentos. A mortalidade perinatal no grupo não monitorizado foi de 19/1000 nascimentos ao passo que a mortalidade fetal após a admissão no serviço foi de 6/1000 nascimentos. Os índices encontrados em nosso material situamse abaixo dos referidos por esses autores.

Krebs e cols. ${ }^{6}$ estudando retrospectivamente 1991 registros cardiotocográficos realizados durante o trabalho de parto entre 1975 e 1977, estabeleceram um escore de FCF para os últimos 30 minutos de monitorização, dando de zero a 2 pontos para a presença de variáveis como linha de base, variabilidade de curta e longa duração (pequenas e grandes oscilações da FCF), acelerações e desacelerações da FCF. Esses autores sugerem que os fetos que apresentam fatores de risco durante o trabalho de parto, mas cuja $\mathrm{FCF}$ permanece sem alterações, comportam-se ao nascimento e período neonatal de maneira similar àqueles que não apresentam tais fatores, e com as mesmas características na cardiotocografia. Da mesma forma, o prognóstico desses fetos é semelhante quando as alterações da FCF aparecem, independente se existem ou não fatores de risco fetal. A incidência de cesáreas emergenciais nos casos monitorizados foi de $24 \%$. Por outro lado quando da presença de sofrimento fetal, e na expectativa de que o parto vaginal ocorra relativamente cedo, os autores o preferem à operação cesariana.

Se de um lado as taxas de partos cesáreos aumentaram no grupo com MFE, às custas de um maior volume de diagnósticos de sofrimento fetal agudo, de outro as indicações por interatividade diminuíram drasticamente. A modificação na incidência das indicações por desproporção céfalopélvica não se deu de forma tão clara como nas duas indicações anteriores. Vários são os fatores que concorrem para o aumento das taxas de operação cesariana em grupos eletronicamente monitorizados durante o parto. Entre eles podemos citar os critérios de interpretação dos registros cardiotocográficos, a experiência do pessoal do serviço e, principalmente, os critérios de valorização das alterações da FCF consignativos de comprometimento fetal. Além desses fatores, as políticas para redução dos índices de cesárea jogam fator importante para a consecução desse objetivo. Tais políticas têm sido implementadas com sucesso em alguns países, mostrando quedas significantes ${ }^{15}$ em particular pela execução de estratégias em gestantes nulíparas em trabalho com progressão ineficiente ou com suspeita diagnóstica de desproporção céfalo-pélvica ${ }^{2,16}$.

\section{Conclusões}

Os resultados do presente estudo permitem concluir que os índices de cesárea mostraram-se superiores no grupo com MFE (38,0\%) quando comparados com os do grupo com AI $(27,2 \%)$ e com os da população geral estudada $(29,9 \%)$; que a principal indicação de cesárea no grupo com AI foi a interatividade $(32,4 \%)$ ao passo que o sofrimento fetal agudo foi a terceira causa (14,3\%); que a principal indicação de cesárea no grupo com MFE foi o sofrimento fetal agudo $(40,6 \%)$ ao passo que a interatividade foi a terceira causa $(10,1 \%)$; que a repercussão da MFE sobre os índices gerais de cesárea foi de apenas $2,7 \%$, o que demonstra não 
ser a MFE um fator determinante para a elevação das taxas de operação cesariana.

\section{SUMMARY}

Electronic fetal heart rate monitoring (EFM) is the most widely used method of direct fetal surveillance especially during labor. In an attempt to elucidate the effect of EFM on cesarean section (CS) rates, a retrospective study was performed at the University Hospital of Santa Maria (HUSM). We studied two groups of patients, consisting of 2114 pregnant women: EFM group $(n=517)$ and intermittent auscultation (IA) group $(n=1597)$. In the EFM group we observed $38.0 \%$ of CS vs. $27.2 \%$ in the IA group. For all patients, the CS rate was $29.9 \%$. Fetal distress was the most common indication for CS in the EFM group (40.6\%), while previous CS was the third cause (10.1\%). On the IA group, fetal distress was the third cause in CS (14.3\%), while previous CS was the most common cause (32.4\%). On the basis of this study, we believe that EFM has no effect in itself on cesarean section rates considering overall deliveries at HUSM. With proper education of the clinician and correct interpretation of the findings, EFM would not increase cesarean section rates, but rather should allow for a more accurate description of intrapartum fetal well-being.

KEY WORDS: Fetal monitoring. Fetal distress. Cesarean section

\section{Referências bibliográficas}

1. Boehm FH, Davidson KK, Barrett JM. The effect of electronic monitoring on the incidence of cesarean section. Am J Obstet Gynecol 1981; 140:295-298.

2. DeMott RK, Sandmire HF. The Green Bay cesarean section study. II. The physician factor as a determinant of cesarean birth rates for failed labor. Am J Obstet Gynecol 1992; 166:1799-1810.

3. Freeman RK, Garite TJ, Nageotte MP. Fetal heart rate monitoring. Willians e Wilkins, 2nd Ed, Baltimore, 1991, p. 21-34.

4. Haverkamp FA, Orleans M, Langerdoerfer S, McFee J, Murphy J, Thompson HE. A controlled trial of the different effects of intrapartum monitoring. Am J Obstet Gynecol 1979; 134:388-412.

5. Kelso IM, Parsons RJ, Lawrence GF, Arora SS, Edmonds DK, Cooke ID. An assessment of continuous fetal heart rate monitoring in labor. Am J Obstet Gynecol 1978; 131:526-532.
6. Krebs HB, Petres RE, Dunn LJ, Smith PJ. Intrapartum fetal heart rate monitoring. VII. The impact of mode of delivery on fetal outcome. Am J Obstet Gynecol 1982; 143:190-194.

7. Mac Donald D, Grant A, Pereira MS, Boylan P, Chalmers I. The Dublin randomized controlled trial of intrapartum fetal heart-rate monitoring. Am J Obstet Gynecol 1985; 152:524-539.

8. Mahomed K, Nyoni R, Mulambo T, Kasule J, Jacobus E. Randomised controlled trial of intrapartum fetal heart rate monitoring. $\mathbf{B r}$ Med $\mathbf{J}$ 1994; 308:497500.

9. Morais EN, Mauad Filho F, Maranhão TMO, Bailão LA. Estudo crítico dos riscos e complicações fetais da monitoragem obstétrica. J Br Ginecol 1981; 91:195-197.

10.Morais EN, Mottecy CS. Associação entre nível e variabilidade de linha de base da freqüência cardíaca fetal com o índice de Apgar. J Br Ginecol 1984; 94:257-262.

11.Morais EN, Mottecy CS. Relação entre desacelerações tardias da freqüência cardíaca fetal durante o parto e a vitalidade do recém-nascido. $\mathbf{J} \mathbf{~ B r}$ Ginecol 1984; 94:337-340.

12.Morais EN, Mottecy CS. Associação entre desaceleração variável da freqüência cardíaca fetal durante o trabalho de parto e o índice de Apgar. J Br Ginecol 1984; 94:167-171.

13.Pello LC, Dawes GS, Smith J, Redman CWG. Screening of the fetal heart rate in early labour. $\mathbf{B r} \mathbf{J}$ Obstet Gynaecol 1988; 95:1128-1136.

14. Renou P, Chang A, Anderson I, Wood C. Controlled trial of fetal intensive care. Am J Obstet Gynecol 1976; 126:470-476.

15. Robson MS, Scudemore IW, Walsh SM. Using the medical audit cycle to reduce cesarean sectio rates. Am J Obstet Gynecol 1996; 174:199-205.

16.Sandmire HF, DeMott RK. The Green Bay cesarean section study. IV. The physician factor as a determinant of cesarean birth rates for the large fetus. Am J Obstet Gynecol 1996; 174:15571564.

17.Taffel SM, Placek PJ, Moien M. 1988 U.S. cesarean section rate at 24,7 per 100 births - a plateau? N Eng1 J Med 1990; 323:199-200.

18.Tutera G, Newman RL. Fetal monitoring: Its effect on the perinatal mortality and cesarean section rates and its complications. Am J Obstet Gynecol 1975; 122:750-754.

19.Zalar, RW, Quilligan EJ. The influence of scalp sampling on the cesarean section rate for fetal distress. Am J Obstet Gynecol 1979; 135:239-246. 